\title{
Evaluating the Feasibility and the Potential Efficacy of e-Learning-Based Speech Therapy (EST) as a Web Application for Speech Training in Dysarthric Patients with Parkinson's Disease: A Case Study
}

Lilian J. Beijer, M.A., ${ }^{1}$ Toni C.M. Rietveld, Ph.D., ${ }^{1,2}$ Vera Hoskam, M.A., ${ }^{1}$ Alexander C.H. Geurts, M.D., Ph.D., ${ }^{1,3}$ and Bert J.M. de Swart, Ph.D. ${ }^{3}$

${ }^{1}$ Department of Research Development and Education, Sint Maartenskliniek, Nijmegen, The Netherlands.

${ }^{2}$ Department of Language and Speech, Faculty of Arts, Radboud University Nijmegen, Nijmegen, The Netherlands.

${ }^{3}$ Department of Rehabilitation, Nijmegen Centre for Evidence Based Practice, Radboud University Nijmegen Medical Centre, Nijmegen, The Netherlands.

\section{Abstract}

Objective: As a Web application for speech training, e-learningbased speech therapy (EST) is assumed to have potential for neurological patients who aim at independent speech training in their home environment. This article reports a case study of a patient with dysarthric speech due to Parkinson's disease (PD) who enrolled in a 4-week intensive speech training through EST. The primary goal was to investigate the feasibility and the potential efficacy of EST as a Web application for speech training in dysarthric patients with PD. Materials and Methods: The participant used EST, following a speech training program containing parts of the pitch limiting voice treatment for patients with PD. The feasibility of EST for independent speech training in the home environment was verified through a questionnaire. The questionnaire addressed the participant's individual experiences with EST as well as the extent of satisfaction with technological features of EST, the content of the speech training, and the suitability of the home training environment. The potential efficacy of EST as a device to improve speech intelligibility was investigated using a repeated measures with randomized blocks design. The proportion of correct orthographic transcriptions of semantically unpredictable sentences as well as ratings of perceived intelligibility on a 10-point scale were used as measures for speech intelligibility. Results: Outcomes of the questionnaire resulted in recommendations to enhance EST feasibility. Speech intelligibility, as measured by transcription scores, improved significantly after EST training. This improvement was maintained for 2 weeks after completing the EST training, whereas considerably lower scores were observed after 11 weeks without training. Subjective ratings of intelligibility did not show significant differences across time. Conclusions: The results of this case study confirm the potential of EST for patients with PD.

Key words: e-health, telehealth, technology

\section{Introduction}

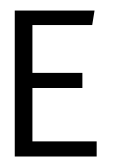

-learning-based speech therapy (EST) is a Web application for speech training (i.e., practice), which is a specific aspect of speech therapy. EST was primarily developed for patients with dysarthric speech following acquired neurological disease such as stroke or Parkinson's disease (PD). ${ }^{1}$ Hence, EST is an example of the increasing number of telehealth applications in speech and language pathology. ${ }^{2} \mathrm{~A}$ vital potential of EST is the possibility to improve speech quality in the absence of a therapist, in a place and at a time of patients' own choice. That is, 
EST provides the opportunity of speech training without spending time, energy, and money traveling to a therapist, thus enhancing access to speech training for neurological patients who tend to be easily fatigued and less mobile due to their physical condition. This independency and easy access to speech training is expected to address the need for intensive speech training in patients with longstanding dysarthria following acquired neurological disorders. ${ }^{3}$ According to our clinical experience, these patients tend to suffer severely from their loss of speech quality, and are highly motivated for intensive speech training. The crucial value of intensive training for neurological patients has been confirmed by research outcomes in the field of motor rehabilitation after stroke. ${ }^{4}$ Also, in speech and language rehabilitation for patients with stroke and PD, intensive training has been proven to be more effective than low frequency training. ${ }^{5,6}$

Moreover, for chronic motor stroke, there are indications that repeated therapy leads to additional improvement of motor activity and even might restore deteriorated quality of motor activity, frequently observed once periods of intensive training have been completed. ${ }^{7}$ Hence, EST might be an excellent adjunct to practice, allowing patients to intensify their speech training. ${ }^{1}$ EST provides a suitable manner for patients with chronic dysarthria to maintain or

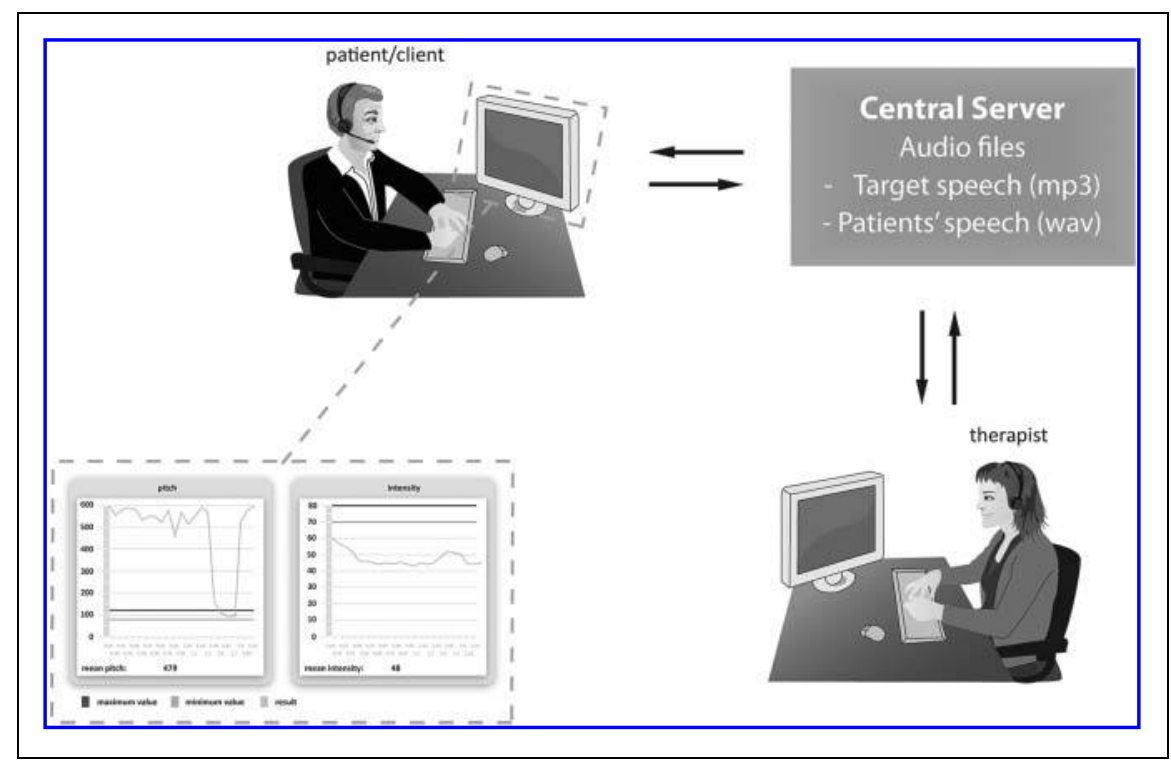

Fig. 1. EST infrastructure, enabling speech therapists to remotely design a tailor-made speech training, and allowing patients to remotely engage the speech training. Patients are provided with automatic visual feedback on their speech intensity and overall pitch. EST, e-learning-based speech therapy. even improve their speech quality without placing high demands on therapy resources. ${ }^{3}$ This might benefit stroke patients as well as patients with PD who finished face-to-face therapy.

In this report, the feasibility and the potential efficacy of EST, containing the pitch limiting voice treatment (PLVT), ${ }^{8}$ is evaluated in a patient with PD. The feasibility to be evaluated in this study addresses the issue whether a patient with PD can deal successfully with the Web-based speech training device EST. In addition, this case study fits in phase I of clinical outcome research. ${ }^{9}$ By exploring the application of this Web device in clinical practice, this study contributes to insight in the potential efficacy of EST. ${ }^{2}$

\section{E-LEARNING-BASED SPEECH THERAPY}

The EST infrastructure is shown in Figure 1. The keystone of this Web application for speech training is a central server. This server contains audio files of target speech as well as audio files from neurological patients who upload their speech through EST. Therapists as well as patients have access to this server. Therapists are allowed to remotely design a tailor-made speech training program by selecting audio files of target speech from the server. Patients enroll in the individual speech training program in their home environment, guided by instructional text to navigate the system and carry out the exercises. By simply pushing a button they record their speech attempts and upload the audio files to the server. For an elaborate description of the EST system, we refer to Beijer et al. ${ }^{1}$

Therapists are provided with a headphone to listen to audio files containing patients' speech and target speech. Patients carrying out the EST training procedure use a headset. As shown in Figure 2, the training procedure for clients contains five basic steps: (1) listening to an audio example of target speech, (2) imitating the target speech, (3) comparing the target with their own speech by means of independent auditory feedback, (4) uploading their speech to the server to obtain additional automatic visual feedback about overall pitch and intensity, and (5) deciding whether or not an adjusted speech attempt should be made to approach the target speech.

\section{Materials and Methods SUBJECT}

A 69-year-old man with PD participated in this study. At the time of inclusion, this patient had 


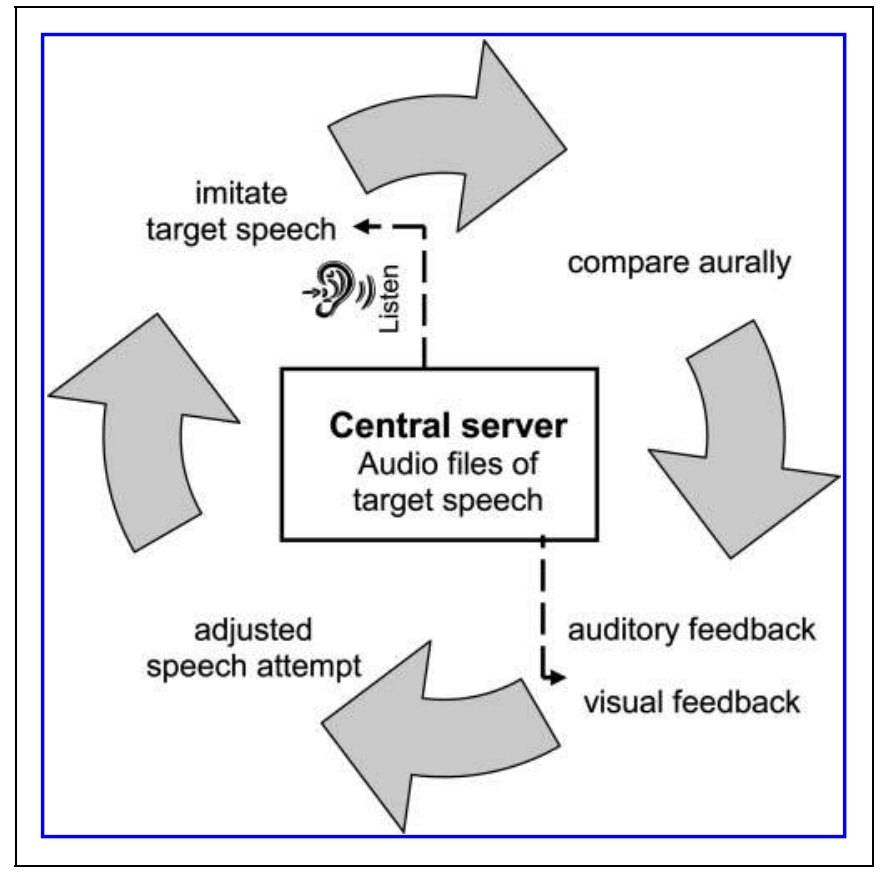

Fig. 2. EST training procedure.

been found to have PD since 11 years. He was assessed at stage 2.5 according to the Hoehn and Yahr ${ }^{10}$ severity grading system, which ranges from 0 to 5. This stage of disease corresponds to symptoms of Parkinsonism on both sides of the body, mild balance problems, and physical independence. Since 2003, the participant had been using the medication Levodopa. No relevant comorbidity was observed.

The participant in our study had an academic degree and was retired as an engineer. He was an active member of the Dutch Parkinson's Disease Association and was actively engaged in various social activities. Four months before our study he completed traditional face-to-face speech therapy, addressing the PLVT for patients with PD. ${ }^{8}$ At the time the participant enrolled in our study, he was experiencing diminished intelligibility in daily conversation.

\section{SPEECH MATERIALS}

For the EST speech training, a PLVT training protocol was set up. The PLVT is a Dutch treatment for PD patients that focuses on improvement of intensity (loudness) and at the same time prevent an increase in vocal pitch and, thereby, in laryngeal muscle tone and laryngeal resistance. By instructing patients to speak "loud and low," the PLVT aims at improvement of speech intelligibility and prevention of strained or pressed high-pitched voicing. ${ }^{8}$ The EST training program contained utterances varying in linguistic complexity from isolated vowels and monosyllabic words with consonant vowel consonant (CVC) structure, up to 10 -word sentences.

For the evaluation of speech intelligibility, a set of 30 semantically unpredictable sentences (SUS) was used. SUS sentences are characterized by a syntactically correct structure in the absence of meaningful semantic context, and are developed to test word intelligibility in sentences. For the purpose of evaluating word intelligibility, the condition of word production in sentences is ecologically more valid than words in isolation (e.g., in word lists). SUS sentences have the advantage of disrupting predictability due to semantic information, ${ }^{11}$ and are frequently used for the evaluation of synthesized speech. ${ }^{12}$ Examples of SUS sentences are (a) the mouth looks at the skin and (b) the house stands on the tree.

In our study, we applied Dutch SUS sentences for the evaluation of intelligibility in dysarthric speech. Each SUS sentence contained four key words (i.e., one verb, two nouns, and one preposition). For an overview, we refer to www.ostt.eu/SUSSentences.aspx.

\section{PROCEDURE}

Assessing auditory discrimination and screening hearing thresholds. Before enrolling in the EST training, the participant performed an auditory discrimination test. This test has been recently developed in The Netherlands to assess auditory discrimination in speech in neurological patients with dysarthria, aiming at speech training through EST. The auditory discrimination test addresses auditory discrimination of variations along five speech dimensions: segmental speech elements (articulation), intensity (loudness), overall pitch, speech rate, and intonation. ${ }^{13}$ The results showed a minimum score of $80 \%$ in all subtests, implying sufficient auditory discrimination abilities for independent auditory feedback required for the EST training. In addition, an audioscreening was carried out, using an Interacoustics AS 208 screening audiometer. The results showed a Fletcher Index of $52 \mathrm{~dB}$ for the right ear and a Fletcher Index of $13 \mathrm{~dB}$ for the left ear, indicating a moderate hearing loss for the right ear and a mild hearing loss for the left ear in the area relevant for speech perception.

EST training. During a 4-week EST period, the participant followed a protocolized PLVT speech training program in his home environment, using a Dell laptop, type Latitude D420, with a 12.1" screen, and a headset Sennheiser PC 131. On the basis of observed intensities and overall pitch in the participant's speech before the EST training period, target values were assigned to these two speech dimensions (i.e., the minimum and the maximum values). The individual targets for our participant were set at mean intensities between 70 and $80 \mathrm{~dB}$ and an 
overall pitch ranging from 105 to $130 \mathrm{~Hz}$. Each week he carried out four subsequent courses containing PLVT exercises, each course being accessible for 1 or 2 days. The participant's access to each course was remotely manipulated by a speech therapist, thus optimizing protocolized speech training. Over the training period, the participant's uploaded speech was remotely monitored by a speech therapist. The therapist and the participant had contact by telephone or by e-mail for additional instructions, when necessary (i.e., once or twice a week).

Speech recordings of SUS sentences. The participant was asked to read aloud a set of 30 SUS sentences at five times across the experimental period: 5 weeks before the EST training (T0), immediately before (T1), and immediately after (T2) the EST training, and finally 2 weeks (T3) and 11 weeks (T4) after completing the EST training. To avoid confounding influences of the participant's medication, to be taken daily at regular times, the recording of SUS sentences also took place at regular times (i.e., between 10.00 AM and 11.00 AM). The speech was recorded digitally on a Fujitsu Siemens laptop with a sampling frequency of $44.100 \mathrm{~Hz}$, using Audacity, version 1.2.6.

Orthographic transcription and intelligibility ratings. For the evaluation of intelligibility, 20 untrained listeners were asked to orthographically transcribe SUS sentences recorded at different times across the experimental period (T0 to T4). Scores were expressed in the proportion correctly transcribed key words per sentence. In addition, the listeners rated the extent of perceived intelligibility for each SUS sentence on a 10-point scale, which is frequently used in the Dutch school system. The scale ranged from " 1 " (extremely bad intelligibility) to "10" (extremely good intelligibility). Ratings below " 6 " indicate an insufficient extent of intelligibility.

A repeated measures randomized blocks design was used with time as a within subject-factor (five levels) and four homogeneous blocks of each five listeners. All blocks contained untrained listeners with an academic degree, differing in age and/or sex. This resulted in four homogeneous blocks containing, respectively, five men between 21 and 30 years old, five women between 21 and 30 years old, five men between 51 and 60 years old and five women between 51 and 60 years old. Within each block, each member was assigned randomly to a set of 30 SUS sentences recorded at one of the five measurement times. The SUS sentences were delivered binaurally by means of a headphone Sennheiser PC131. Each sentence was presented only once. Using the E-prime software tool, version $1.2,{ }^{14}$ the listeners were allowed to carry out the transcription task at their own pace.
Questionnaire. To obtain qualitative information addressing individual experiences using EST, the participant was asked to fill in a questionnaire once the EST training was completed. The questionnaire addressed the participant's experiences with technologyrelated aspects throughout the EST training procedure, as well as the participant's individual condition in using EST. In addition, he was asked to rate the extent to which he was satisfied with (1) the technological features of EST, (2) the content of the speech training program (PLVT) in EST, and (3) the home training environment during the EST training. A 10-point scale, ranging from "1" (extremely unsatisfied) to "10" (extremely satisfied'), was applied for these ratings. Ratings below " 6 " indicate an insufficient extent of satisfaction. Throughout the questionnaire, the participant had the opportunity to report additional personal comments. Finally, the participant was invited to recommend improvements for EST.

\section{Results}

\section{ORTHOGRAPHIC TRANSCRIPTIONS}

As shown in Figure 3, the orthographic transcription scores were considerably higher immediately after the EST training, whereas a lowering of scores was observed 2 weeks after completing the training period. This lowering of transcription scores, representing diminished intelligibility, proceeds in the course of time without speech training after the EST period.

An analysis of variance for repeated measures was carried out on the scores obtained in the orthographical transcriptions. Adopting a 0.05 significance level, the within-subject factor time was significant: $F_{2.068,6.204}=10.471, p=0.010$, Huynh-Feldt corrected. The effect size $\left(\eta_{\mathrm{p}}{ }^{2}\right)$ was 0.777 . Paired comparisons showed a significant higher transcription score after the EST training (T2), with a mean score of 0.89 (standard deviation $[S D]=0.045$ ) compared to a mean

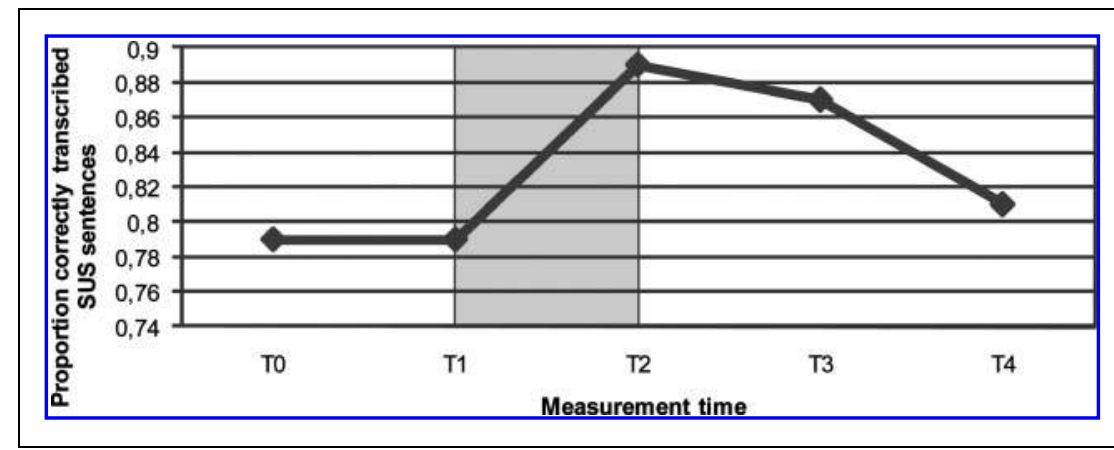

Fig. 3. Mean proportion of correctly transcribed semantically unpredictable sentences across time. The EST training period is marked by a shaded block. 


\section{BEIJER ET AL.}

score of 0.79 (SD = 0.029) immediately before the EST training (T1). Paired comparisons between other measurement times did not yield statistically significant differences. However, a tendency of lowering transcription scores is observed 2 weeks after the EST training (at T3): a slightly lower mean score occurred, implying slightly diminished intelligibility. This tendency proceeded by further deterioration of intelligibility 11 weeks after completing the EST training (T4). At this point, the mean transcription score approached the scores before the training period, at T0 and T1. Apparently, 11 weeks without training resulted in loss of the achieved improvement during the EST period.

\section{INTELLIGIBILITY RATINGS}

Figure 4 shows the mean intelligibility ratings on a 10-point scale across time. Obviously, a pattern slightly different from the transcription scores occurred. Whereas the orthographic transcriptions achieved the highest mean score immediately after the EST training (T2), the intelligibility ratings at this point were slightly lower than before the training. At $\mathrm{T} 2$ the mean rating was $5.3(\mathrm{SD}=1.01)$ and at $\mathrm{T} 1$ the mean was $5.7(\mathrm{SD}=0.55)$. Two weeks after the EST training, the highest mean rating was observed $(M=6.4, \mathrm{SD}=0.80)$.

An analysis of variance for repeated measures on the intelligibility ratings did not yield a significant within-subject factor time $\left(F_{2.400,7.200}=3.026, p=0.107\right.$, Huynh-Feldt corrected, observed power $=0.438$ ).

\section{QUESTIONNAIRE}

With respect to technological aspects of the EST training procedure, slight technological problems in the first week were reported, mainly addressing the consequences of disruptions in the Internet

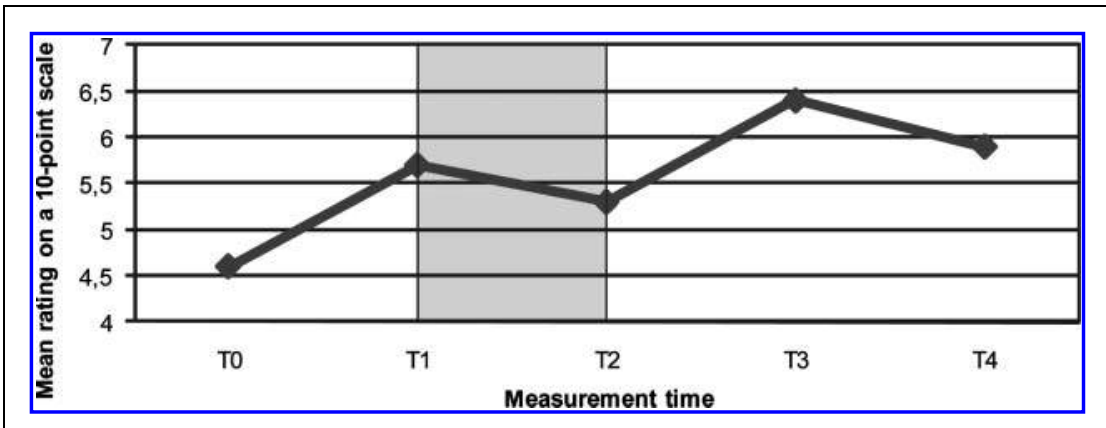

Fig. 4. Mean intelligibility ratings across time. 1, lowest value; 10, highest value. Ratings below 6 represent insufficient intelligibility. The EST training period is marked by a shaded block. connection. No problems were mentioned with logging into the patient account, navigating the system, accessing the exercises in the speech training program, carrying out the training procedure, or uploading speech to the server. Experiences with EST, related to the participant's individual physical and cognitive condition, resulted in a recommendation of a laptop screen larger than $12.1^{\prime \prime}$ to facilitate reading the instructional text and the training items. With respect to the speech training program, restriction of warming up exercises was recommended to avoid premature fatigue during the genuine speech exercises.

The results of the satisfaction ratings are presented in Table 1 . Ratings about technological features in EST achieved a mean of 6.67 (2.35) on a 10-point scale. Items addressing the content of the speech training program (PLVT) achieved a mean rate of 7.57 (0.98), and the possibility of a home training environment was qualified with the highest mean rate of 8.33 (1.55).

From the ratings in Table 1, it becomes obvious that the size of the laptop screen urgently required improvement. The item "adjustments of the speech training program" addresses the therapist's possibility to remotely vary the content of the training program. That is, after the first 3 weeks of training, our participant reported considerable fatigue carrying out all exercises per course in the training program, impeding repeated speech attempts per item. Therefore, the therapist reduced the number of exercises per course in the fourth week, resulting in a considerably higher satisfaction rating on the item addressing the number of exercises per course. The items related to the home training environment were highly appreciated on the rating scale.

Additional to the questionnaire and the satisfaction ratings on a 10-point scale in Table 1, the participant was allowed to comment on his personal experiences with EST. Although he reported to find EST an attractive device for independent speech training in his home environment, he recommended additional contact once a week with a therapist by telephone or through videoconferencing. The participant also advised improvement of the user interface to enhance EST attractiveness for users. Further, although the home training environment was highly appreciated, ergonomic requirements for using EST should be taken into account. In addition, the keyboard and the mouse should be adapted to meet the requirements of patients with $\mathrm{PD}$, who tend to suffer from diminished motor coordination. Finally, although satisfied with the graphs for visual feedback on speech intensity and overall pitch for his personal use, our participant recommended adjustment of the 
Table 1. Ratings of the Extent of Satisfaction Addressing Technological Features of e-Learning-Based Speech Therapy, the Content of the Speech Training Program, and the Home Training Environment

RATING

EXTENT OF SATISFACTION (10-POINT SCALE)

$\left.\begin{array}{l|l}\hline \text { Technological features } & 7 \\ \hline \text { 1. Ease of using EST } & 8 \\ \hline \text { 2. Training procedure (manner of exercising) } & 7 \\ \hline \begin{array}{l}\text { 3. Attractiveness of EST as a speech training } \\ \text { device }\end{array} & 1 \\ \hline \text { 4. Size of laptop screen } & 6 \\ \hline \text { 5. Clarity of instructions to navigate in EST } & 8 \\ \hline \text { 6. Auditory feedback } & 9 \\ \hline \text { 7. Visual feedback } & 8 \\ \hline \begin{array}{l}\text { 8. Suitability of feedback as a cue to improve } \\ \text { speech }\end{array} & 6 \\ \hline \text { 9. Robustness to technical disturbances } & 7 \\ \hline \text { Speech training program } & 8 \\ \hline \text { 10. Instructional text throughout EST training } & 8 \\ \hline \text { 11. Type of speech exercises } & 8 \\ \hline \text { 12. Degree of difficulty in speech exercises } & 7 \\ \hline \text { 13. Design of the speech training program } \\ \text { throughout the EST training period }\end{array}\right)$

1, lowest value; 10, highest value. Ratings below 6 represent insufficient satisfaction.

EST, e-learning-based speech therapy. visual feedback to avoid problems in patients less experienced in the interpretation of visual graphs.

\section{Discussions}

The results of our study show that speech practice through EST has the potential to improve speech intelligibility. Apparently, the Web application EST facilitates easy access to speech training, thus enhancing the opportunity to intensify training. The observed improvement of speech quality through intensive Web-based training in our case study underscores the value of practice. This is in agreement with research findings in the field of motor rehabilitation as well as speech and language rehabilitation after stroke, showing that intensive training is effective and improves therapy results. ${ }^{4,7}$ As indicated by our case study, the benefit of intensive training may also apply to patients with chronic dysarthria due to PD. Indeed, the results of EST showed statistically significant higher transcription scores immediately after completing this Web-based speech training.

Two weeks after completing EST, a slight decay of the achieved improvement occurred, and even dramatically lower transcription scores were observed on the long term (i.e., 11 weeks after the EST training period). This decay of skills over time underscores the transient nature of the improvement. Therefore, EST may not only improve speech intelligibility, but also help to maintain the achieved speech quality in patients with chronic dysarthria. ${ }^{3}$

In contrast to the orthographic transcription scores, the ratings on perceived intelligibility on a 10-point scale did not show statistically significant effects across time. With somewhat lower ratings immediately after completing the EST training, the pattern of mean ratings of perceived intelligibility throughout the experimental period actually differed from that of the orthographic transcriptions. It might be argued that for these subjective ratings, in the absence of anchor stimuli, the interrater agreement tends to be lower due to raters' personal anchors for valuation of speech intelligibility. ${ }^{15}$ Since we used a randomized blocks design with repeated measures, the distinct pattern of perceived intelligibility ratings across the experimental period might be caused by such differences in personal anchors. The observed difference between orthographic transcription scores and the intelligibility ratings might imply that subjective rating is not a suitable measure to evaluate speech intelligibility.

In the context of patient-centered healthcare, qualitative information addressing the patient's experiences with EST was obtained by means of a questionnaire. The participant in our study was able to operate the EST device successfully. He highly appreciated the possibility of independent speech training in his home environment, although additional contact with a speech therapist by telephone or 


\section{BEIJER ET AL.}

videoconferencing was preferred. Yet, various adaptations of the EST equipment might be required to enhance suitability for neurologically impaired patients in general. These findings are in line with previously reported advantages and disadvantages of telerehabilitation. ${ }^{16}$

Applying recommendations of the American Telemedicine Association, ${ }^{17}$ future research should address technical, clinical, human, and ergonomic factors of EST. In addition, economic analyses should be conducted to establish the cost effectiveness of this Web application for speech training. In the near future, we will investigate user preferences for traditional face-to-face speech training versus Webbased training in patients with acquired dysarthria. Since this patient group is heterogeneous with respect to background variables such as sex, age, underlying disease, severity of dysarthria, computer experience, and physical condition, it is vital to detect background profiles of potential EST users. This might avoid disappointing experiences or even premature abandonment of this Web application for speech training.

For now, the results of this case study confirm the potential efficacy of EST in patients with PD. Our findings contribute to establishing sound fundamentals for research into the efficacy of EST to improve and maintain speech quality in larger groups of patients. ${ }^{9}$

\section{Acknowledgments}

The authors thank Bert van Esterik for participating in this case study, Marijn van Beers for his technical assistance during the experimental period, and Margo van Stiphout for her assistance with the figures in this article.

\section{Disclosure Statement}

No competing financial interests exist.

\section{REFERENCES}

1. Beijer $L$, Rietveld $T$, van Beers $M$, Slangen $R$, van den Heuvel $H$, de Swart $B$, Geurts A. E-learning based speech therapy (EST): A web application for speech training. Telemed J E Health 2010;16:177-180.

2. Mashima PS, Doarn CR. Overview of telehealth activities in speech-language pathology. Telemed J E Health 2008;14:1101-1117.

3. Palmer $\mathrm{R}$, Enderby $\mathrm{P}$, Hawley $\mathrm{M}$. Addressing the needs of speakers with longstanding dysarthria: Computerized and traditional therapy compared. Int J Lang Commun Disord 2007;42(Suppl 1):61-79.

4. Kwakkel G, Wagenaar RC, Twisk JWR, Lankhorst GJ, Koetsier JC. Intensity of leg and arm training after primary middle-cerebral-artery-stroke: A randomised trial. Lancet 1999;354:191-196.
5. Ramig LO, Sapir S, Countryman S, Pawlas AA, O'Brien C, Hoehn M, et al. Intensive voice treatment (LSVT) for patients with Parkinson's disease: A 2 year follow-up. J Neurol Neurosurg Psychiatry 2001;71:493-498.

6. Teasell RW, Kalra L. What's new in stroke rehabilitation? Advances in Stroke 2003. Stroke 2004;35:383-385.

7. Rijntjes $M$, Haevernick $K$, Barzel $A$, van den Bussche $H$, Ketels $G$, Weiller $C$. Repeat therapy for chronic motor stroke: A pilot study for feasibility and efficacy. Neurorehabil Neural Repair 2009;23:275-280.

8. de Swart BJM, Willemse SC, Maassen BAM, Horstink MWIM. Improving of voicing in patients with Parkinson's disease by speech therapy. Neurology 2003;60:498-500.

9. Robey RR, Schultz MC. A model for conducting clinical-outcome research: An adaptation of the standard protocol for use in aphasiology. Aphasiology 1998; 12:787-810.

10. Hoehn M, Yahr M. Parkinsonism: Onset, progression and mortality. Neurology 1967;17:427-442.

11. Jekosch U. Voice and speech quality perception. Assessment and evaluation. Berlin Heidelberg: Springer-Verlag; 2005:117-118.

12. Benoit $C$, Grice M, Hazan V. The SUS test: A method for the assessment of textto-speech synthesis intelligibility using semantically unpredictable sentences. Speech Commun 1996;18:381-392.

13. Beijer L, Rietveld T, van Stiphout A. Auditory discrimination as a condition for e-learning based speech therapy (EST): A proposal for assessment of auditory discrimination in dysarthric patients. (Submitted).

14. Schneider W, Eschman A, Zuccolotto A. E-prime reference guide. Pittsburg: Psychology software tools, 2002.

15. Rietveld T. Subjective measuring in speech pathology. (In preparation).

16. Torsney K. Advantages and disadvantages of telerehabilitation for persons with neurological disabilities. Neurorehabilitation 2003;18:183-185.

17. Krupinski E, Dimmick S, Grigsby J, Mogel G, Pkin D, Speedy S, et al. Policy: Research Recommendations for the American Telemedicine Association. Telemed J E Health 2006;12:579-589.

Address correspondence to: Lilian J. Beijer, M.A. Department of Research Development and Education Sint Maartenskliniek P.O. Box 9011 6500 GM Nijmegen The Netherlands

E-mail: 1.beijer@maartenskliniek.nl

Received: December 24, 2009

Revised: February 1, 2010

Accepted: February 1, 2010 
This article has been cited by:

1. L.J. BEIJER, A.C.M. RIETVELD, A. VAN STIPHOUT. 2011. Auditory discrimination as a condition for E-learning based Speech Therapy: A proposal for an Auditory Discrimination Test (ADT) for adult dysarthric speakers. Journal of Communication Disorders . [CrossRef] 